ARTICLE

Received 29 Oct 2015 | Accepted 19 Jan 2017 | Published 13 Mar 2017

DOI: $10.1038 /$ ncomms14664

OPEN

\title{
Sfrp5 identifies murine cardiac progenitors for all myocardial structures except for the right ventricle
}

\author{
Masayuki Fujii', Akane Sakaguchi ${ }^{2,3}$, Ryo Kamata ${ }^{1}$, Masataka Nagao ${ }^{4}$, Yutaka Kikuchi ${ }^{5}$, Silvia M. Evans ${ }^{6}$, \\ Masao Yoshizumi ${ }^{1}$, Akihiko Shimono ${ }^{7, \dagger}$, Yumiko Saga ${ }^{2,3,8} \&$ Hiroki Kokubo ${ }^{1}$
}

Upon acquirement of pulmonary circulation, the ancestral heart may have been remodelled coincidently with, or accompanied by, the production and rearrangement of progenitor cells. However, the progenitor populations that give rise to the left ventricle (LV) and sinus venosus (SV) are still ambiguous. Here we show that the expression of Secreted frizzled-related protein Sfrp5 in the mouse identifies common progenitors for the outflow tract (OFT), $\mathrm{LV}$, atrium and SV but not the right ventricle (RV). Sfrp5 expression begins at the lateral sides of the cardiac crescent, excluding early differentiating regions, and continues in the venous pole, which gives rise to the SV. Lineage-tracing analysis revealed that descendants of Sfrp5-expressing cells at E7.5 contribute not only to the SV but also to the LV, atria and OFT and are found also in the dorsal splanchnic mesoderm accompanied by the expression of the secondary heart field marker, Islet1. These findings provide insight into the arrangement of cardiac progenitors for systemic circulation.

\footnotetext{
${ }^{1}$ Department of Cardiovascular Physiology and Medicine, Graduate School of Biomedical and Health Sciences, Hiroshima University, 1-2-3 Kasumi, Minamiku, Hiroshima 734-8551, Japan. ${ }^{2}$ Department of Mammalian Development, National Institute of Genetics, Mishima $411-8540$ Japan. ${ }^{3}$ The Graduate University for Advanced Studies (SOKENDAI), Hayama, Kanagawa 240-0193, Japan. ${ }^{4}$ Department of Forensic Medicine, Graduate School of Biomedical and Health Sciences, Hiroshima University, 1-2-3 Kasumi, Minamiku, Hiroshima 734-8551, Japan. ${ }^{5}$ Department of Biological Science, Graduate School of Science, Hiroshima University, 1-3-2 Kagamiyama, Higashi-hiroshima 739-8511, Japan. ${ }^{6}$ Department of Medicine, School of Medicine UC San Diego, 9500 Gilman Drive, La Jolla, California 92093, USA. ${ }^{7}$ Center for Developmental Biology (CDB), RIKEN Kobe, 2-2-3 Minatojima-minamimachi, Chuou-ku, Kobe 650-0047, Japan. ${ }^{8}$ Department of Biological Sciences, Graduate School of Science, The University of Tokyo, Hongo 7-3-1, Bunkyo-ku, Tokyo 113-0033, Japan. $\dagger$ Present address: Bristol-Myers K.K. 6-5-1 Nishi-Shinjuku, Shinjuku-ku, Tokyo 163-1328, Japan. Correspondence and requests for materials should be addressed to Y.S. (email: ysaga@nig.ac.jp) or to H.K. (email: hkokubo@hiroshima-u.ac.jp).
} 
$\mathrm{M}$ ammalian cardiac development begins with the cardiac crescent, which is composed of a bilateral cardiogenic splanchnic mesoderm, and the progressive differentiation of progenitors into cardiomyocytes to form the primary heart tube. During the successive development of the four-chambered heart, two independent pulmonary and systemic circulatory systems are established. In this process, the production and rearrangement of progenitor cells may account for remodelling of the ancestral heart into these two separate systems. Recent findings pertaining to another cardiac lineage called the secondary heart field (SHF) modified the classical view of cardiac development that the myocardium was derived from a single source. The SHF emerges in the splanchnic mesoderm to supply cells at both the arterial and venous poles of the heart tube, including the right ventricle (RV), most of the atria and outflow tract $(\mathrm{OFT})^{1-3}$. However, the progenitor population that forms the first heart field (FHF), which gives rise to the left ventricle (LV) and the rest of the atria, and the sinus venosus (SV) are still ambiguous.

SHF progenitor cells, which transiently express Islet1 (Isl1), have been shown to express cardiac markers, including Fibroblast growth factor 10 (Fgf10), Myocyte enhancer factor 2c (Mef2c), T-box transcription factor 1 (Tbx-1), as well as other markers, and contribute to the RV, the OFT and most of the atria ${ }^{1,2,4-9}$. FHF progenitor cells have yet to be defined because the FHF lineage, which subsequently gives rise to the LV and a subset of the atria, is recognized only after the expression of differentiation markers such as Myosin light chain $2 a(\mathrm{Mlc2a})$ and NK-2 transcription factor related, locus 5 (Nkx2-5) in the cardiac crescent ${ }^{3,10,11}$.

The SV is a cavity formed by a thin cardiac muscular wall, which drains blood from systemic veins and the coronary sinus into the right atrium, and gives rise to the sinoatrial node at the joint to the superior cardinal vein during embryogenesis. The origin of the SV has been previously addressed and mapped to the lateral rim of the heart fields in chickens by dye injection experiments ${ }^{12}$. Recently, the cell population in the lateral rim of the heart fields, which was defined by the expression of $m u t G 5 / h s p 68 / L a c Z$ in mice, was found to contribute to the $\mathrm{SV}^{13}$. The venous pole was reported to be the precursor of the SV, which is marked by the expression of Tbx18 from E8.25 (refs 13,14). Lineage-tracing experiments with Tbx18-Cre mice revealed that Tbx18-expressing cells form the SV and proepicardium ${ }^{15,16}$. Thus the origin of the SV has been suggested to be in the caudolateral regions of the cardiac crescent, although its relation to the FHF or SHF remains unclear.

Wnt signalling is one of the most important regulatory pathways in vertebrate cardiogenesis ${ }^{17-21}$. Wnt antagonism by Dkk1 or Crescent induces heart formation in vertebrates ${ }^{22-24}$, and Dkk1 is required for head induction in mammals ${ }^{25}$. Secreted frizzled-related protein (Sfrp) 1 and Sfrp2, which are also Wnt inhibitors, are expressed in the heart and are involved in cardiac tissue repair ${ }^{26,27}$, while the expression pattern and function of another subfamily member, Sfrp5, during cardiac development are unknown.

In this report, we show that Sfrp5 expression is detected in lateral and caudal regions of the cardiac crescent, is expressed in cells distinct from those positive for markers of differentiated cardiomyocytes and the SHF and continues to be expressed in the developing SV. However, descendants of Sfrp5-expressing cells notably contribute to cardiomyocytes in all chambers of the heart, except for the RV. These observations suggest that Sfrp5-expressing cells in the lateral region of the cardiac crescent are progenitor cells for the OFT, the LV, the left and right atria and the SV. Hyperplasia in the inflow tract (IFT) was observed in $S f r p 1 / 2 / 5$ triple knockout (TKO) mouse embryos, suggesting that Sfrp5 regulates the proliferation of cardiac progenitor cells in the venous pole.

\section{Results}

Sfrp5 is expressed in progenitor cells of the $\mathrm{SV}$ and epicardium. Wnt signalling is required for myocardial induction and is implicated in several steps of heart development ${ }^{17-21}$. The Sfrp subfamily genes (Sfrp1,2 and 5) are known to encode secreted decoy receptors for Wnt signalling ${ }^{28}$ and to participate in the maintenance of adult cardiac function ${ }^{26,29}$. Among the three Sfrp genes, we found that Sfrp5 was specifically expressed in a particular part of the cardiac crescent at E7.5. Sfrp5 was detected in regions lateral and caudal to the region of Mlc2a and Nkx2-5 expression, which marks differentiating cardiomyocytes (Fig. 1a,b). The expression area of $S f r p 5$ was clearly distinct from those of Mlc2a and Nkx2-5 (Supplementary Fig. 1). During the heart tube looping stage (E8.0-E8.5), the expression of Sfrp5 was observed continuously in the venous pole of the heart (Fig. 1c-e), the pattern of which was distinct from Mlc2a and $N k \times 2-5$, indicating that the Sfrp5-expressing area excludes differentiating cardiomyocytes. At E9.5, Sfrp5 expression was confined to the ventral venous pole, which is known to contain cardiac progenitors for the SV as well as the epicardium, suggesting that Sfrp5 might be expressed in such progenitors. To investigate this possibility, we performed double fluorescent immunohistochemistry using Tbx18 and WT1, specific markers for the SV and epicardium, respectively. To monitor the Sfrp5 expression, we used Sfrp5-venusYFP embryos. At E9.5, yellow fluorescent protein (YFP) was detected strongly in the ventral side and weakly in the dorsal side of the venous pole, similar to the pattern of Sfrp5 expression (Fig. 1f). The YFP signal highly overlapped with Tbx18 staining and moderately with WT1 staining (Fig. 1f,g), suggesting that Sfrp5 is expressed in progenitor cells of the SV and the epicardium.

Cardiomyocytes in the SV show a unique gene expression profile, that is, TroponinT (TnT)- and Hcn4-positive but Nkx2-5negative. This differs from the Nkx2-5- and TnT-positive chamber myocardium ${ }^{30}$. To further examine the expression profile of Sfrp5-venusYFP-expressing cells, we conducted marker expression analysis. At E9.5, Sfrp5-venusYFP-expressing cells were negative for Nkx2-5, TnT and Hcn4 (Supplementary Fig. 2a), whereas, at E10.5, these cells expressed TnT and Hon4 but not Nkx2-5 (Supplementary Fig. 2b). These results indicate that Sfrp5-expressing cells in the ventral venous pole at E9.5 represent progenitors for the SV and that they differentiate into cardiomyocytes in the SV at E10.5. Thus the Sfrp5-expressing area at E7.5 may be the precursor of the SV.

Sfrp5 is expressed in progenitors of cardiac components except for RV. We next determined whether progenitors of the SV correspond to Sfrp5-expressing cells in the cardiac crescent. To test this possibility, we generated a Sfrp5-Cre line (Supplementary Fig. 3) and investigated the lineages of $S f r p 5$-expressing cells by crossing this line with Rosa26R or CAG-floxed-CAT-eGFP reporter mice ${ }^{31}$. At E8.5, $\beta$-galactosidase (LacZ) staining was unexpectedly observed in the entire heart tube, except for the most rostral portion (Fig. 2a). At E9.5, LacZ staining was observed in the OFT, LV, atria and venous pole but not in the RV (Fig. 2b). Labelled cells overlapped with TnT in the OFT, LV and atria, suggesting that descendants of Sfrp5-expressing cells differentiated into chamber myocardium in addition to the SV (Fig. 2c). At E13.5, LacZ-stained cells mostly occupied not only the LV but were also found in the WT1-expressing pericardium (Fig. 2d,e) and the cluster of differentiation 31 (CD31)- or platelet endothelial cell adhesion molecule-expressing endocardium (Fig. 2f). Labelled cells were found in part of the interventricular septum and rarely in the RV (Fig. 2b,d) and those that were found in the $\mathrm{RV}$ tended to localize in the 


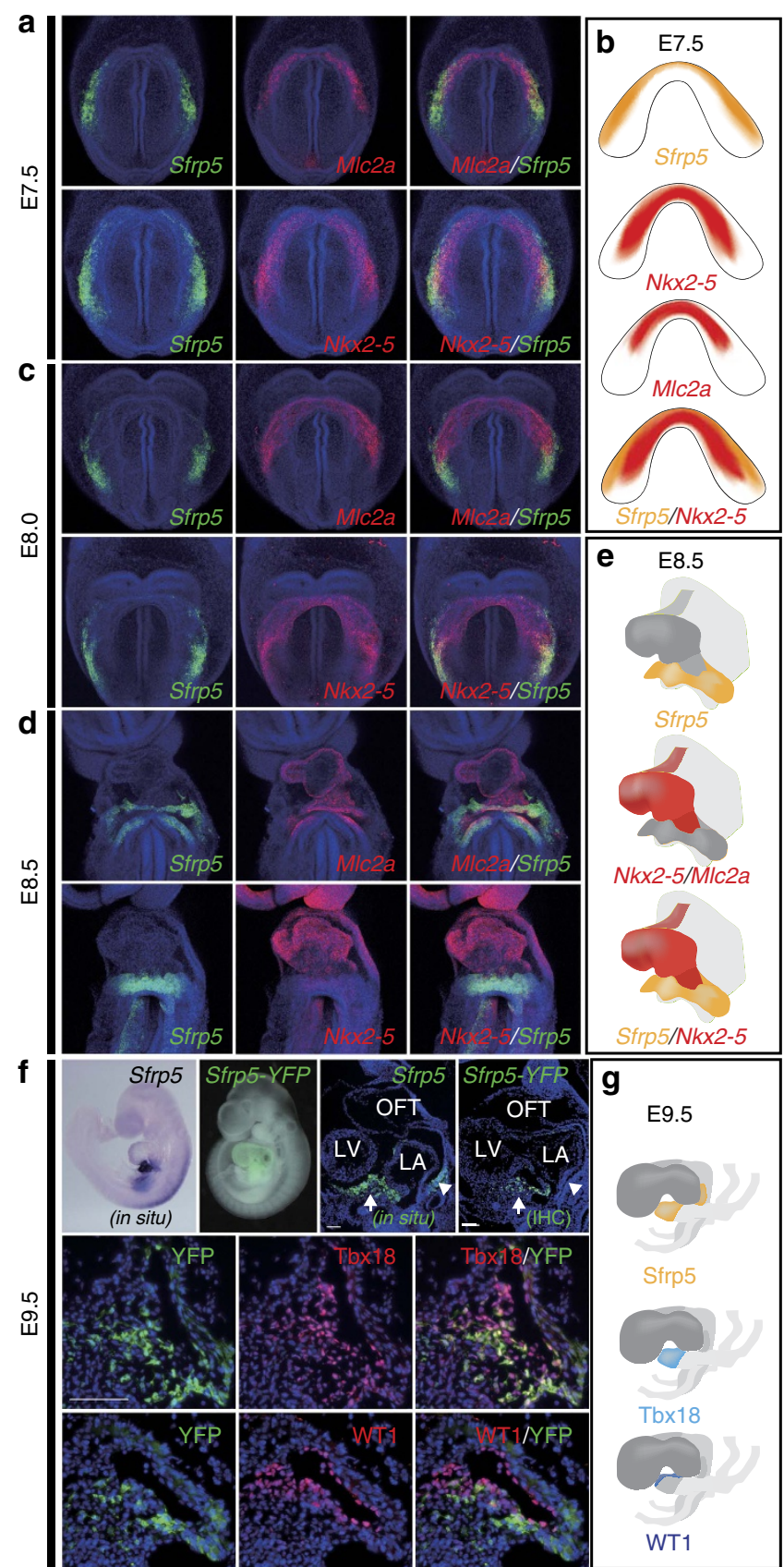

Figure 1 | Sfrp5 is expressed in the lateral cardiac crescent and subsequently in the SV. (a,c,d) Double fluorescent whole-mount ISH of mouse embryos at E7.5 (a), E8.0 (c) and E8.5 (d) for detection of Sfrp5 (green) using Mlc2a or Nkx2-5 (red) as indicated in each panel. $(\mathbf{b}, \mathbf{e}, \mathbf{g})$ Schematic drawings of gene expression $(\mathbf{b}, \mathbf{e})$ and protein distribution (g). (f) Whole-mount ISH (in situ), YFP image merged with bright field image (Sfrp5-YFP), section in situ with probe for Sfrp5 and single or double immunohistochemistry (IHC) of Sfrp5-venusYFP KI embryos using anti-GFP antibodies with anti-Tbx18 or anti-WT1 antibodies at E9.5, as indicated in each panel. Scale bars $=$ Scale bars, $50 \mu \mathrm{m}$.

trabecular myocardium (Fig. 2f). These observations suggest that Sfrp5 is expressed in progenitors of the SV, pericardium, epicardium and endocardium, as well as in all chamber myocardium except for the RV. Given that Isl1 lineages contribute to all myocardium except for the $\mathrm{LV}^{32,33}$, the fate of Isl1 lineages to the RV or Sfrp5 lineages to the LV may be exclusively determined during the earliest stage of cardiogenesis.
Since Sfrp5 expression is confined to the venous pole of the heart at E8.5, which does not include chamber myocardium, we speculated that cells expressing Sfrp5 prior to E8.5 might include progenitors of the FHF. To evaluate the fate of Sfrp5-expressing cells at specific developmental stages, we generated a tamoxifeninducible (Estrogen Receptor T2 (ERT2)) Cre mouse line (Supplementary Fig. 3). After crossing Sfrp5-ERT2Cre mice with reporter mice, Sfrp5-expressing cells were labelled at E7.5 via tamoxifen injection and analysed at E10.5. LacZ staining revealed that cells labelled at E7.5 were found in the OFT, LV, atria, $\mathrm{SV}$ and venous pole but not in the RV (Fig. 3a,b). Interestingly, labelled cells were still found in the atria at E8.5 and E9.5 (Supplementary Fig. 4), indicating that Sfrp5-expressing cells at the venous pole may supply cardiomyocytes for the IFT. These data suggest that Sfrp5-expressing cells at E7.5 include common progenitors for the SV, OFT and all chamber myocardium except for the RV. There is therefore a strong possibility that Sfrp5 expression marks progenitors of the FHF.

Sfrp5 derivatives contribute to the FHF and part of the SHF lineages. To test this possibility, the expression of Sfrp5 was compared with that of the FHF marker genes, Hcn4 and Tbx5. Hcn4 is expressed in the prospective LV at E7.5, with expression shifting towards the $\mathrm{SV}^{30,34}$. Tbx5 is expressed in the FHF lineage at E6.5-E7.5 (refs 35-37). The expression of Hcn4 was observed in the anteromedial region compared with $S f r p 5$, while region marked by $T b x 5$ overlapped with and extended anteriorly from that marked by Sfrp5 (Fig. 3c,d). Since the expression of Hcn 4 was restricted to the anterior portion of the $T b \times 5$ expression area, we concluded that Hcn4 might mark only the early differentiated FHF lineage. Conversely, Tbx5 marks both undifferentiated and differentiating FHF progenitors, among which Sfrp5 expression is restricted to undifferentiated FHF progenitors existing in the lateral sides of the cardiac crescent. Moreover, cells weakly expressing YFP in the primitive ventricle and IFT were co-distributed with HCN4 in the Sfrp5-venusYFP mouse embryo at E8.5 (Fig. 3e), suggesting that descendants of Sfrp5expressing cells contribute to the FHF lineage.

Lineage analysis also revealed that Sfrp5-expressing cells at E7.5 contributed to the OFT and atria, indicating that these cells also include progenitors of the SHF. However, at E7.5, the expression pattern of Sfrp5 was distinct from that of Isl1, an SHF marker, although some overlap was found in the expression in the caudal region (Fig. 3c,d). At E9.5, Isl1 transcripts and protein were expressed in the splanchnic mesoderm, including the dorsal venous pole, but were rarely expressed in the ventral venous pole, which contains Sfrp5-expressing cells (Supplementary Figs 5 and 6). To clarify whether the expression of Sfrp5 precedes that of Isl 1 in the splanchnic mesoderm, we conducted lineage-tracing analysis using Isl1-Cre and CAG-floxed-CAT-mRFP reporter/ Sfrp5-venusYFP mice. We found that the YFP signal in the ventral venous pole rarely overlapped with derivatives of Isl1-expressing cells (Fig. 3f). In Sfrp5-Cre/CAG-floxed-CAT-eGFP-reporter mice, however, GFP-labelled cells expressed Isl 1 and Fgf10 in the dorsal splanchnic mesoderm and part of the OFT (Fig. $3 \mathrm{~g}$ and Supplementary Fig. 7). These findings suggest that derivatives of Sfrp5-expressing cells also contributed to part of the SHF lineage, which may give rise to the OFT and atria. Notably, these GFP-labelled cells were rarely found in the anterior splanchnic mesoderm, where Tbx1, which is known to also be expressed in the $\mathrm{SHF}^{38,39}$, was distributed (Fig. 3g).

Sfrp5-YFP-expressing ES cells differentiate into cardiac components. We next tested whether Sfrp5-venusYFP-expressing embryonic stem (ES) cells could differentiate into cardiac 
a

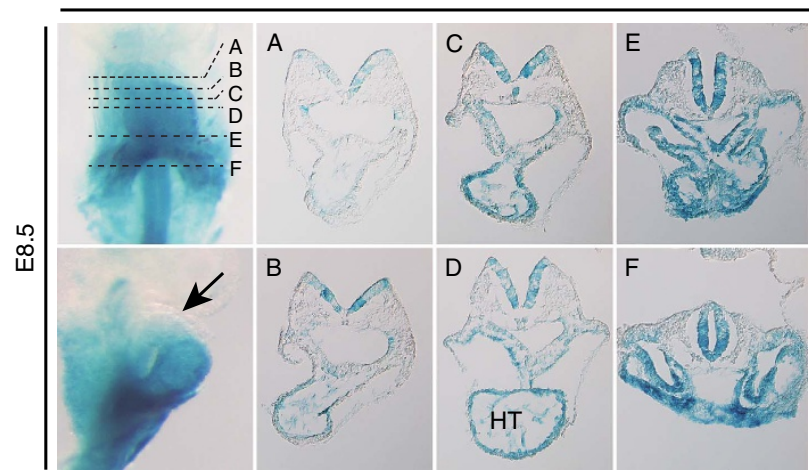

b

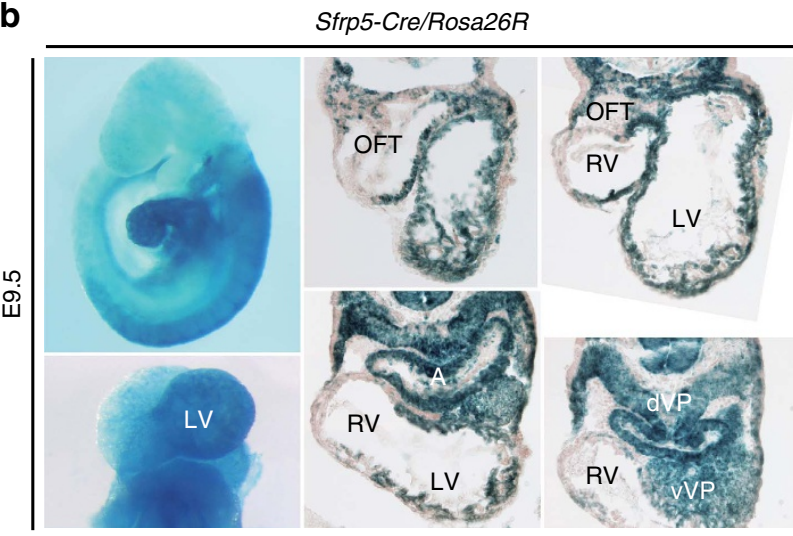

C

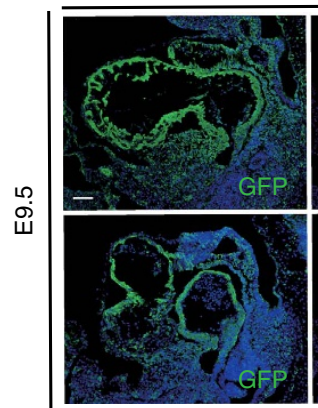

Sfrp5-Cre / CAGCATeGFP

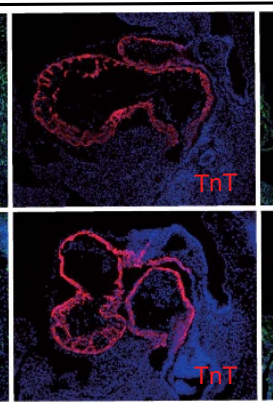

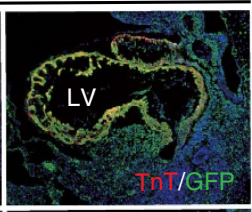

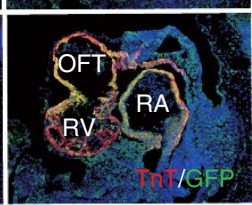

d

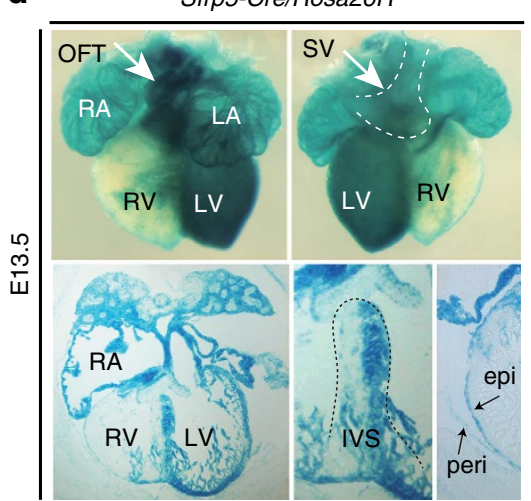

$\mathbf{e}$

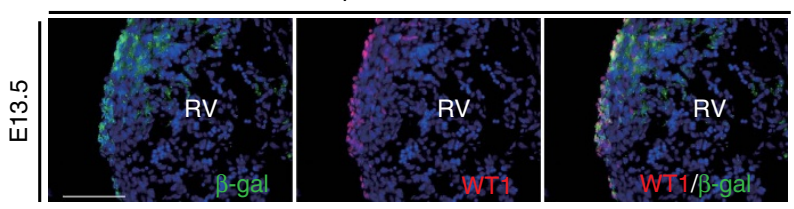

f

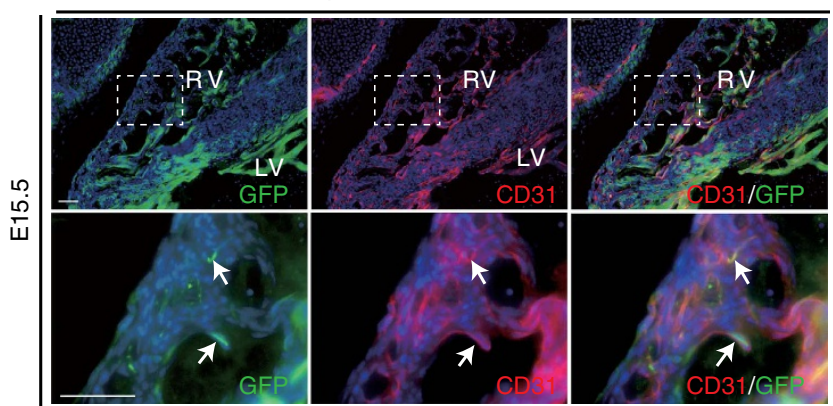

Figure 2 | Descendants of Sfrp5-expressing cells contribute to the SV and all chamber myocardium except for the RV. (a,b,d) Whole-mount and section LacZ staining of Sfrp5-Cre/Rosa26R embryos at E8.5 (a), E9.5 (b) and E13.5 (d). HT: heart tube; dVP: dorsal venous pole; vVP: ventral venous pole; epi: epicardium; peri: pericardium, IVS; interventricular septum. (c,e,f) Double immunohistochemistry of Sfrp5-Cre/CAG-floxed-CAT-eGFP (c,f) or Sfrp5-Cre/ Rosa26R (e) embryos at E 9.5 (c), E 13.5 (e) and E15.5 (f), using anti-GFP or anti- $\beta$-gal (green) antibodies with anti-TnT, anti-Isl1, anti-WT1 or anti-CD31 antibodies (red). Rectangles in upper panels (f) are magnified in lower panels. Arrows indicate cells co-expressing YFP and CD31 (f). Scale bars $=50 \mu \mathrm{m}$.

components, such as endothelial, smooth muscle and myocardial cells. On day 2, the peak of YFP expression was found to be ahead of those of other differentiation markers (Supplementary Fig. 8). On day 8, CD31-, $\alpha$-smooth muscle actin- and TnT-positive cells were found in the cell mass (Supplementary Fig. 8), suggesting that Sfrp5 could also serve as a marker gene of precursor cells for cardiac components in an in vitro differentiation system.

Sfrps may not be involved in cardiac differentiation. Our results imply that $S f r p 5$ is expressed and retained in cardiac progenitors but lost upon their differentiation, indicating that the loss of Sfrp5 might enhance the differentiation of these cardiac progenitors. To test this possibility, we generated Sfrp1/2/5 TKO mice. As reported previously, only TKO mice show severe growth retardation due to the compensatory functions of Sfrp1 and Sfrp2 (ref. 40). We focussed our analysis on the cardiac phenotype and analysed several lineage markers via in situ hybridization (ISH) at E9.5, the latest stage at which TKO embryos can be collected. Similar to controls, Nkx2-5 was present in the heart tube but not in the venous pole in TKO embryos (Fig. 4a). This indicates that the differentiation of cardiomyocytes occurred normally until this stage. The expression of Tbx5, Isl1 and Tbx18 was normal (Fig. 4a), suggesting that formation of the FHF, SHF and SV was not affected. These observations suggest that Sfrps may not affect differentiation.

Sfrps prevent proliferation by inhibiting canonical Wnt signalling. TKO embryos appeared to have an enlarged IFT, as compared with the OFT. To determine whether Sfrps might regulate the 
a

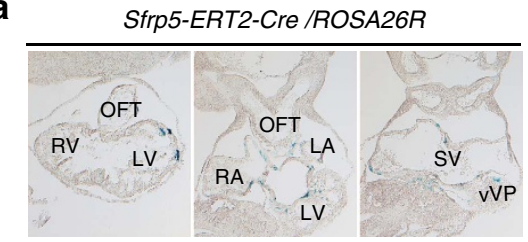

(Labelled at E7.5 and detected at E10.5)

b
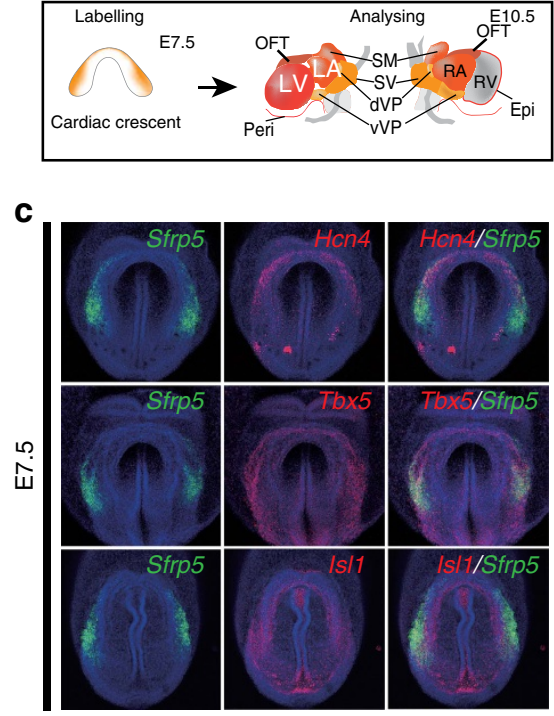

d

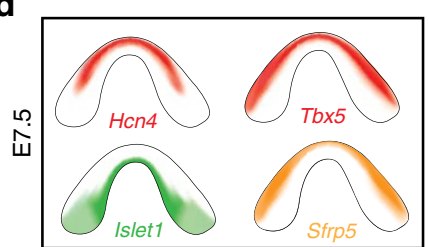

e

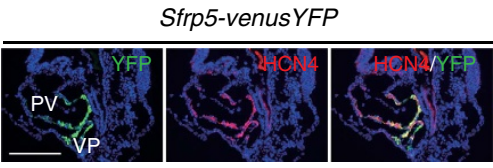

$\mathbf{f}$

Sfrp5-venusYFP/Is/1-Cre/CAGCATmRFP

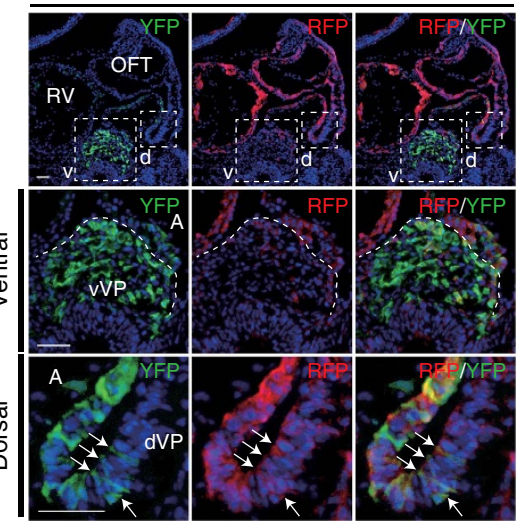

g

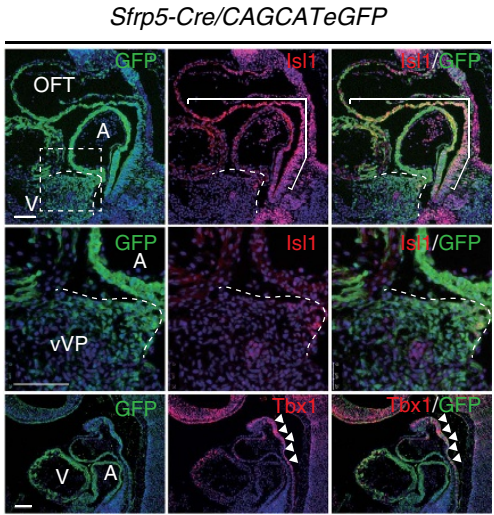

Figure 3 | Sfrp5-expressing cells at E7.5 contribute to the SV and all chamber myocardium except for the RV. (a) Representative photographs of LacZ-stained sections from Sfrp5-ERT2Cre/Rosa-lacZ embryos at E10.5, labelled by tamoxifen at E7.5. (b) Schematic drawing for the expression of Sfrp5 (orange) and the cardiac contribution of its lineages (red) when cells were labelled at E7.5 and analysed at E10.5. (c) Double fluorescent whole-mount ISH of mouse embryos at E7.5 for detection of Sfrp5 (green) and Hcn4, Tbx5 or Is/7 (red), as indicated in each panel. (d) Schematic drawings of gene expression of Sfrp5, Is/1, Hcn4 and Tbx5. (e) Double immunohistochemistry of Sfrp5-venusYFP at E8.5 using anti-GFP (green) and anti-HCN4 (red) antibodies. Co-expression with HCN4 was found in the VP. Weak expression of YFP also co-distributed with HCN4-positive cells in the IFT and part of the primitive ventricle (PV). (f,g) Double immunohistochemistry of Sfrp5-venusYFP (e), Sfrp5-venusYFP/Is/7-Cre/CAG-(floxed)-CAT-mRFP (f) or Sfrp5-Cre/CAG-(floxed)-CAT-eGFP (g) embryos at E9.5 using anti-GFP (green) antibodies and anti-HCN4, anti-RFP, anti-Isl1 or anti-Tbx1 (red) antibodies. Rectangles in upper panels are magnified in lower panels ( $\mathrm{v}$ : ventral; $\mathrm{d}$ : dorsal). Co-expression with Isl1 was detected (arrows in panel (f) and parenthesis in panel $(\mathbf{g})$ ). Boundaries for the VP from the atrium are indicated by white dotted lines $(\mathbf{f}, \mathbf{g})$. Signal for Tbx1, but not GFP, was observed in the anterior splanchnic mesoderm and anterior OFT (arrowheads in panel (g)). A, atrium.

proliferation of cardiac progenitors, we next assessed whether Wnt/ $\beta$-catenin signalling and progenitor cell proliferation in the venous pole were affected in TKO embryos. In control embryos, $\beta$-catenin accumulated in the ventricles and atria, but less $\beta$-catenin was observed in YFP-positive cardiac progenitor cells in the ventral venous pole (Fig. 4b). In TKO embryos, however, $\beta$-catenin accumulated even in YFP-positive cardiac progenitor cells (Fig. 4b). In control mice, Ki67 was more highly expressed in YFP-negative cells than in YFP-positive cells, suggesting that proliferation may be inhibited in Sfrp5-expressing cardiac progenitors (Fig. 4c,d). In contrast, the percentage of Ki67-positive cells in TKO embryos increased in YFP-positive cells, as compared with the percentage in control samples (Fig. 4c,d). These results indicate that Sfrps prevent the proliferation of cardiac progenitors by inhibiting canonical Wnt signalling.

\section{Discussion}

Based on our findings, we propose a model for the contribution of Sfrp5-expressing cardiac precursors to heart development, except for the RV (Supplementary Fig. 9). We found that Sfrp5-expressing cells at E7.5 contributed not only to the OFT, LV and atria and also to the SV by fate mapping experiments using Sfrp5-Cre and-ERT2Cre mice. This observation gives rise to the possibility that the FHF lineage and the SV might share a common origin. This idea has been implied previously by the expression and lineage-tracing experiments of Hcn4, because Hcn4-expressing cells ultimately yield most of the LV, portions of the atria and the cardiac conduction system ${ }^{30,41-44}$. We found that the expression of Sfrp5 rarely overlapped with that of Hcn4, while cells weakly expressing YFP in the PV and IFT at E8.5 largely overlapped with Hcn4. The expression of Sfrp5 also 
a

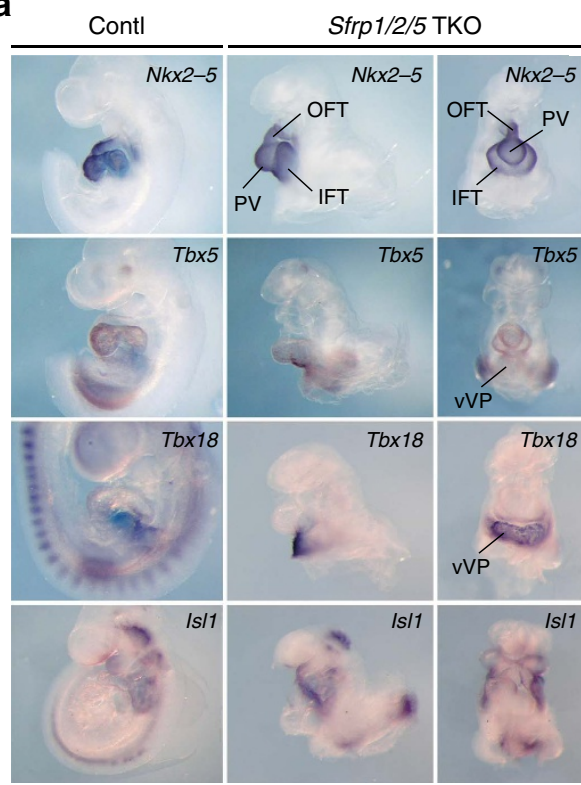

b

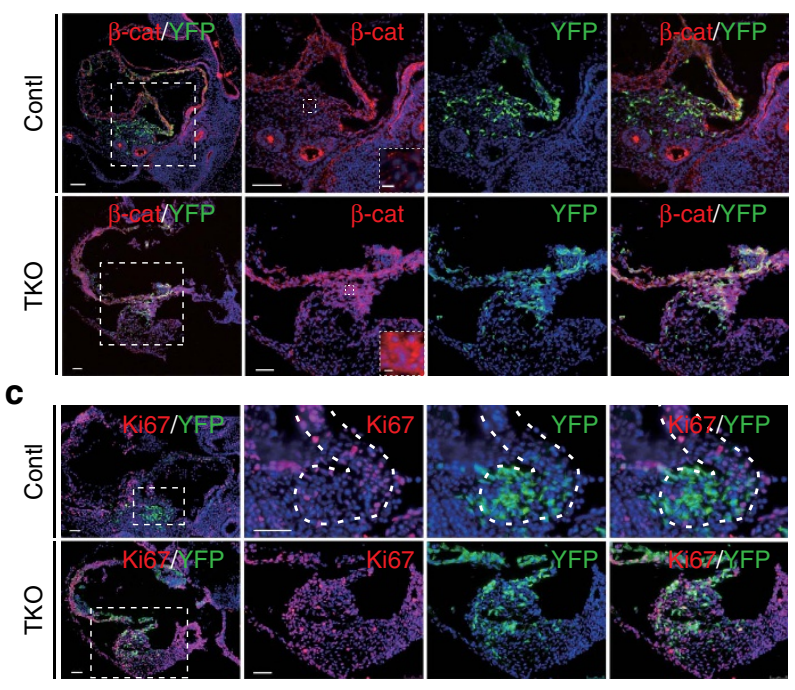

Average (\%) of Ki67+

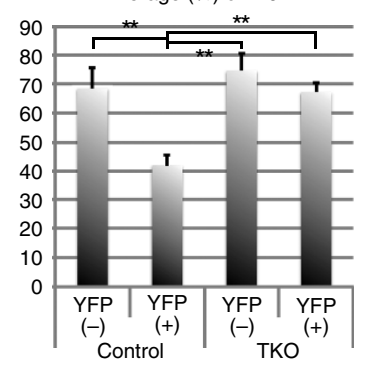

Figure 4 | Sfrp1/2/5 TKO embryos exhibit hyperplasia of the IFT accompanied by activated canonical Wnt signalling. (a) ISH of control and TKO embryos with probes for Nkx2-5, Tbx5, Tbx18 and Is/1 genes. PV: primitive ventricle. (b,c) Double immunohistochemistry using anti- $\beta$-catenin (b) or anti-Ki67 (c) antibodies in control and TKO embryos. Rectangles from the left end panels are magnified in the right panels. Further magnified photos, located in the right bottom window, show that $\beta$-catenin accumulated in YFP-expressing cells in the ventral venous pole in TKO embryos. (d) Percentage of Ki67 positive $(+)$ cells in Sfrp5-YFP-expressing $(+)$ or negative $(-)$ cells in control and TKO embryos $(n=3)$. Data are presented as mean \pm s.d.; ${ }^{\star \star} P<0.01$, as compared with control samples. Scale bars $=50 \mu \mathrm{m}(5 \mu \mathrm{m}$ in dotted rectangles $)$.

largely overlapped with that of $T b x 5$ at E7.5, and early labelling of Tbx5-expressing cells at E6.5-E7.5 are reported to mark cells that are predominantly restricted to the LV and atria ${ }^{36,37}$, suggesting that Sfrp5-expressing cells include progenitors for the FHF. Moreover, Sfrp5 was continuously expressed in cells giving rise to the SV, suggesting that cells that transiently express Sfrp5 contribute to the FHF. Sfrp5-expressing cells start to express $T b \times 18$ at around E8.0, and forced expression of Tbx18 reportedly promotes differentiation of cells into the SV, including the conduction system ${ }^{45}$, and represses a subset of myocardial genes $^{46}$. Thus Sfrp5-expressing cells could maintain the potential of cells to differentiate into both the SV and FHF until Tbx18 expression initiates.

Alternatively, the early Sfrp5-expressing progenitor pool might already be heterogeneous in the commitment to each differentiation program. Since Tbx5-expressing cells at E6.5-E7.5 would not contribute to the OFT (personal communication from Dr Yashiro), a precise comparison of expression domains and lineages of Sfrp5 and Tbx5 may discriminate Sfrp5-expressing progenitors that contribute to the OFT from those that contribute to other regions in the cardiac crescent. However, the expression domain of Sfrp5 determined by ISH could be more restricted than it actually is at early stages, given the possibility of Cre recombination occurring in Sfrp5-expressing progenitors at levels undetectable by ISH. Thus the timing for cell fate determination and the potential for Sfrp5-expressing progenitor cells to differentiate should be addressed in future.

Another observation was that a portion of Sfrp5-expressing cells later expressed Isl 1 and contributed to the OFT and atria (Fig. 5). This suggests that the Isll-expressing SHF progenitor population has at least two distinct origins, one of which is derived from Sfrp5-expressing cells. We also found that Tbx1 is expressed in the anterior splanchnic mesoderm in which descendants of Sfrp5-expressing cells did not appear, indicating that $T b \times 1$ could mark the subpopulation of Isl1-expressing cells in the SHF. Moreover, lineages of Sfrp5-expressing cells did not contribute to the RV. It was previously reported that mesodermal cells of the first branchial arch are involved in $\mathrm{RV}$ formation and that $T b x 1$ is expressed in the first branchial arch and descendants of Tbx1-expressing cells contribute to the $\mathrm{RV}^{38,39,47-49}$. These findings suggest a distinct origin of the $\mathrm{RV}$ relative to that of the other chambers. Given that septated ventricles have been evolutionally acquired to separate systemic and pulmonary circulation ${ }^{50}$, our results provide new insight into the arrangement of cardiac progenitors before the evolutionary emergence of the RV.

The role of Wnt signalling in cardiac development has been extensively reported ${ }^{17}$. The hyperactivation of $\mathrm{Wnt} / \beta$-catenin signalling in cardiac precursors leads to enhanced proliferation and inhibition of further differentiation ${ }^{51,52}$. In our study, 

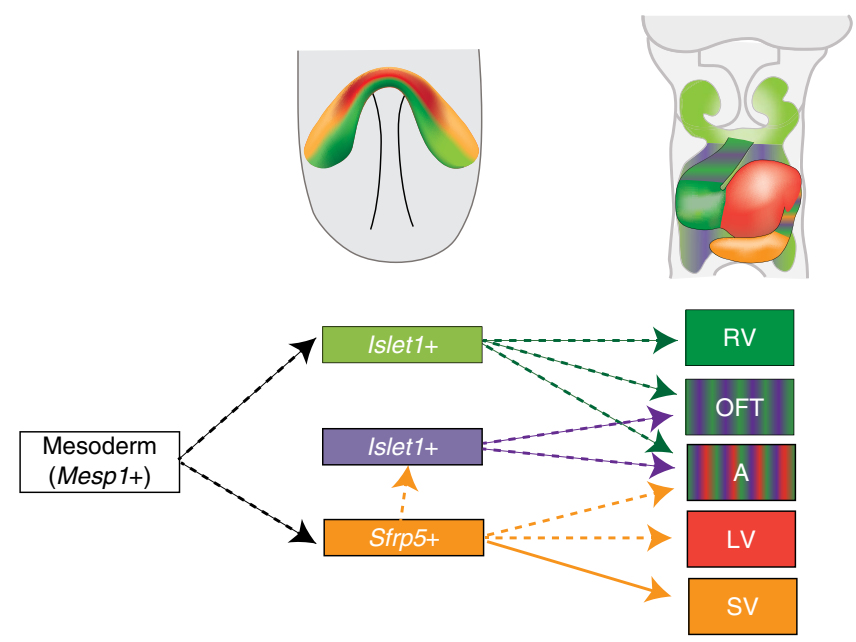

Figure 5 | Model for the contribution of Sfrp5-expressing cardiac precursors to heart development. Upper panels show the expression area of Sfrp5 (orange) and Islet1 (light green) and the distribution of descendants of Sfrp5-expressing (red) and Islet7-expressing (green) cells at E7.5 (right) and E9.5 (left). The lower panel shows the lineage contribution to each part of the heart. Mesodermal cells, which transiently express Mesp1, may become cardiac precursor cells expressing Islet1 (light green) or Sfrp5 (orange). A portion of Sfrp5-expressing cells later begins to express Islet1 (purple). Transient Islet1-expressing cells that do not express Sfrp5 contribute to the RV, OFT and atrium (green), and cells that express Sfrp5 contribute to the OFT and atrium (purple) but not to the RV. Cells that transiently express Sfrp5 contribute to the LV, atrium and OFT (orange), and cells that continuously express Sfrp5 form the SV.

increased proliferation was observed in TKO embryos, but differentiation into primitive cardiomyocytes was not impaired. We speculate that artificial activation and lack of inhibition of $\mathrm{Wnt} / \beta$-catenin signalling may result in different outcomes during myocardial differentiation, and a primary role of $\mathrm{Wnt} / \beta$-catenin signalling in this context may be the regulation of proliferation rather than differentiation. Since Sfrp 1/2/5 has been reported to regulate planar cell polarity pathways through the non-canonical Wnt pathway ${ }^{40}$, tissue- or cell-specific functions of Sfrps may exist. Further understanding of the molecular basis of cardiac progenitor differentiation into various types of cardiomyocytes could provide new therapeutic insights for regenerative medicine.

\section{Methods}

Mice. To generate Sfrp5-Cre recombinase (Cre) and Sfrp5-modified ERT2 conjugated with Cre knock-in mouse lines, a strategy similar to that used to generate the Sfrp5-venusYFP mouse line was adopted ${ }^{40}$. Targeting vectors were constructed by using a cassette containing Cre-or ERT2Cre-recombinase genes with frt-PGK-neomycin (neo)-bpA-frt, which was ligated with KpnI-SmaI and SpeI$\mathrm{XbaI}$ fragments for long and short homologous arms, respectively.

A MC1-DTA-negative selection marker cassette was also added to the outside of the short homologous arm to enrich for homologous recombinants

(Supplementary Fig. 3). For recombination of the targeting vector for ERT2Cre, the CRISPR-Cas9 system was used. A 5'-GGCCAGGAGTACGACTACTACGG-3' sequence was inserted into the pX330 plasmid (Addgene) using the BbsI site and was then co-transfected with the targeting vector. Homologous recombination was confirmed by southern hybridization for the Sfrp5-Cre line, using XbaI-EcoRI fragments for the probe, and by PCR for the Sfrp5-ERT2Cre line, using the following primer set: Neo6: $5^{\prime}$-CGACCACCAAGCGAAACATC- $3^{\prime}$ and SAcontRR9: 5'-CCCAGGGAAGGGAATTGTTCTAG-3'. Deletion of Neo by crossing with the ROSA-Flp mouse line was confirmed by PCR, using the following primer set: DeltaNeoF1: $5^{\prime}$-CCTACATGCGCCCACTAGCCGT- $3^{\prime}$ and DeltaNeoR2: $5^{\prime}$-CCTAGCGACCCCAAGCATCC-3' ${ }^{\prime}$. TKO embryos were obtained by crossing with $S f r p 1 / 2 / 5 ;-I-,+1-,-1-$ or $-/-,+/-$, venus $(\mathrm{v}) / \mathrm{v}^{40}$. Isll-Cre mouse lines have been previously generated using gene-targeting techniques ${ }^{7}$. The ROSA26R mouse line was a gift from Dr Soriano ${ }^{31}$. These lines were maintained in a 129 and C57BL/6 mixed background. A CAG-floxed-CATlyn-mRFP mouse line was newly and CAG-floxed-CAT-eGFP mouse line was previously established using transgenic techniques using $\mathrm{C} 3 \mathrm{HeJ} \times \mathrm{C} 57 \mathrm{BL} / 6 \mathrm{~J}$ F1 hybrid mice ${ }^{53}$.

Tamoxifen injection. Tamoxifen (Sigma) was injected peritoneally into 8-20-week-old pregnant female mice at a dosage of $35 \mathrm{mg} \mathrm{kg}^{-1}$ at E7.5-E9.5. Embryos were dissected on E10.5 or E13.5 and subjected to lacZ staining.

Whole-mount double fluorescent ISH. Embryos were dissected between E7.5 and E9.5 and fixed with 4\% paraformaldehyde in PBS for durations of $1 \mathrm{~h}$ to overnight. After dehydration using a methanol series, embryos were rehydrated with $0.1 \%$ Tween 20 in PBS (PBST), bleached with $6 \% \mathrm{H}_{2} \mathrm{O}_{2}$ in PBS, treated with Protease $\mathrm{K}$ and then hybridized with digoxygenin (DIG)- and/or dinitrophenyllabelled probes at $68^{\circ} \mathrm{C}$ overnight. They were then washed with $5 \times$ and $2 \times \mathrm{SSC} /$ $50 \%$ formamide, PBST and blocking reagent for $1 \mathrm{~h}$. For detection of DIG-labelled probes, embryos were incubated with anti-DIG antibodies and detected using a TSA-Alexa555 detection system according to the manufacturer's instructions. For detection of dinitrophenyl, TSA-Alexa488 was used. Embryos were analysed by confocal laser microscopy (Leica LSM 5 PASCAL system).

Immunohistochemistry and LacZ-staining. Embryos were embedded with optimum cutting temperature compound after fixation and were sectioned at 10-14 $\mu \mathrm{m}$ increments. After washing with PBS and $1 \mathrm{~h}$ of preincubation with $1 \%$ BSA in PBS for blocking, sections were incubated with $500 \times$ diluted primary antibodies (anti-GFP: Nakalai GF090R or Abcam ab290; anti-Tbx1: Santa Cruz sc-17874; anti-Tbx18: Santa Cruz SC17869; anti-Wt1: Abcam ab10670; anti-Nkx2-5: Abcam ab35842; anti-TnT: Thermo MS-295-P0; anti-Hcn4: Alomone Labs APC052; anti-RFP: MBL PM005; anti- $\beta$-catenin: Millipore 05-665; anti-Ki67: Abcam ab15580) at $4{ }^{\circ} \mathrm{C}$ overnight. The sections were then incubated with $500 \times$ diluted secondary antibodies conjugated with Alexa Fluor 488 or 555 (Molecular Probes). Nuclei were counterstained with 4,6-diamidino-2phenylindole. For LacZ-staining, frozen sections were incubated with X-gal solution at room temperature overnight after washing with PBS.

Cardiac induction of ES cells. ES cells were isolated from blastocysts of Sfrp5-venusYFP mice and maintained in Leukemia Inhibitory Factor-containing $\left(1,000 \mathrm{U} \mathrm{ml}^{-1}\right) \mathrm{ES}$ culture medium (DMEM with $10 \%$ fetal bovine serum, $1 \times$ Non-Essential Amino Acids Solution, $1 \mathrm{mM}$ sodium pyruvate, and $10^{-4} \mathrm{M}$ 2-mercaptoethanol). For cardiac induction, embryoid bodies, which were formed by the hanging-drop culture method for $48 \mathrm{~h}$, were plated in 2-well chamber slides or 98-well plates (Nunc) and cultured for 8 days in ES culture medium without Leukemia Inhibitory Factor.

Real-time PCR. Total RNA was isolated using TRIzol (Invitrogen). Reverse transcription was performed with the ReverTra Ace qPCR RT Kit (TOYOBO). Real-time PCR was conducted using SYBR Premix Ex Taq II (Takara Bio Inc. or Kapa Biosystems Inc.). Intensities of PCR products were measured and analysed using Mini-Opticon (Bio-Rad). The following primers were used: YFP: forward $5^{\prime}$-CCTGGTCGAG CTGGACGGCGAC- $3^{\prime}$ and reverse $5^{\prime}$-TCACGAACTCCA GCAGGACCATG-3', Nkx2-5: forward $5^{\prime}$-GCTACAAGTGCAAGCGACAG- $3^{\prime}$ and reverse $5^{\prime}$-GGGTAGGCGTTGTAGCCATA- $3^{\prime}, \beta$-MHC: forward $5^{\prime}$-TCCA CGGGGAAGAGCATCCAT- $3^{\prime}$ and reverse $5^{\prime}$-CAGACTCTGGAGGCTCTTC ACT, Hcn4: forward 5'-CTCAACTCAGGCGTCTTCAAC- $3^{\prime}$ and reverse $5^{\prime}$-CCACCGAAGTAGTAGCAGCAG-3', and G3pdh: forward 5'-ACCACAGTCC ATGCCATCAC- $3^{\prime}$ and reverse $5^{\prime}$-TCCACCACCCTGTTGCTGTA $-3^{\prime}$. Amplification conditions were $5 \mathrm{~s}$ at $95^{\circ} \mathrm{C}, 20 \mathrm{~s}$ at $60^{\circ} \mathrm{C}$ and $15 \mathrm{~s}$ at $72^{\circ} \mathrm{C}$ for 49 cycles. G3pdh was amplified as an internal control.

Statistical analysis. A parametric multiple comparison test, one-way analysis of variance with Tukey-Kramer post hoc analysis, was mainly used (unless otherwise indicated) when Bartlett's test $(P<0.05)$ showed homogeneity of variances with the Ekuseru-Toukei 2012 software (Social Survey Research Information Co., Ltd.). All data are expressed as mean \pm s.d. $P<0.05$ was considered statistically significant.

Data availability. The authors declare that all data supporting the findings of this study are available within the paper and its Supplementary Information files or available on reasonable request from the corresponding authors.

\section{References}

1. Moorman, A. F., Christoffels, V. M., Anderson, R. H. \& van den Hoff, M. J. The heart-forming fields: one or multiple? Philos. Trans. R. Soc. Lond. B Biol. Sci. 362, 1257-1265 (2007).

2. Buckingham, M., Meilhac, S. \& Zaffran, S. Building the mammalian heart from two sources of myocardial cells. Nature Rev. Genet. 6, 826-835 (2005). 
3. Abu-Issa, R. \& Kirby, M. L. Heart field: from mesoderm to heart tube. Annu. Rev. Cell Dev. Biol. 23, 45-68 (2007).

4. Viragh, S. \& Challice, C. E. Origin and differentiation of cardiac muscle cells in the mouse. J. Ultrastruct. Res. 42, 1-24 (1973).

5. Kelly, R. G., Brown, N. A. \& Buckingham, M. E. The arterial pole of the mouse heart forms from Fgf10-expressing cells in pharyngeal mesoderm. Dev. Cell 1, 435-440 (2001).

6. Mjaatvedt, C. H. et al. The outflow tract of the heart is recruited from a novel heart-forming field. Dev. Biol 238, 97-109 (2001).

7. Cai, C. L. et al. Isll identifies a cardiac progenitor population that proliferates prior to differentiation and contributes a majority of cells to the heart. Dev. Cell 5, 877-889 (2003).

8. Sun, Y. et al. Islet 1 is expressed in distinct cardiovascular lineages, including pacemaker and coronary vascular cells. Dev. Biol. 304, 286-296 (2007).

9. Prall, O. W. et al. An Nkx2-5/Bmp2/Smad1 negative feedback loop controls heart progenitor specification and proliferation. Cell 128, 947-959 (2007).

10. Snarr, B. S., Kern, C. B. \& Wessels, A. Origin and fate of cardiac mesenchyme. Dev. Dynam. 237, 2804-2819 (2008).

11. Evans, S. M., Yelon, D., Conlon, F. L. \& Kirby, M. L. Myocardial lineage development. Circ. Res. 107, 1428-1444 (2010).

12. Stalsberg, H. \& DeHaan, R. L. The precardiac areas and formation of the tubular heart in the chick embryo. Dev. Biol. 19, 128-159 (1969).

13. Mommersteeg, M. T. et al. The sinus venosus progenitors separate and diversify from the first and second heart fields early in development. Cardiovasc. Res. 87, 92-101 (2010).

14. Christoffels, V. M. et al. Formation of the venous pole of the heart from an Nkx2-5-negative precursor population requires Tbx18. Circ. Res. 98, 1555-1563 (2006).

15. Cai, C. L. et al. A myocardial lineage derives from Tbx18 epicardial cells. Nature 454, 104-108 (2008)

16. Christoffels, V. M. et al. Tbx18 and the fate of epicardial progenitors. Nature 458, E8-E9 discussion E9-E10 (2009).

17. Brade, T., Manner, J. \& Kuhl, M. The role of Wnt signalling in cardiac development and tissue remodelling in the mature heart. Cardiovasc. Res. 72, 198-209 (2006)

18. Cohen, E. D., Tian, Y. \& Morrisey, E. E. Wnt signaling: an essential regulator of cardiovascular differentiation, morphogenesis and progenitor self-renewal. Development 135, 789-798 (2008).

19. Gessert, S. \& Kuhl, M. The multiple phases and faces of Wnt signaling during cardiac differentiation and development. Circ. Res. 107, 186-199 (2010).

20. Martin, L. K. et al. Canonical WNT signaling enhances stem cell expression in the developing heart without a corresponding inhibition of cardiogenic differentiation. Stem Cells Dev. 20, 1973-1983 (2012).

21. Ueno, S. et al. Biphasic role for Wnt/beta-catenin signaling in cardiac specification in zebrafish and embryonic stem cells. Proc. Natl Acad. Sci. USA 104, 9685-9690 (2007).

22. Marvin, M. J., Di Rocco, G., Gardiner, A., Bush, S. M. \& Lassar, A. B. Inhibition of Wnt activity induces heart formation from posterior mesoderm. Genes Dev. 15, 316-327 (2001).

23. Schneider, V. A. \& Mercola, M. Wnt antagonism initiates cardiogenesis in Xenopus laevis. Genes Dev. 15, 304-315 (2001).

24. Tzahor, E. \& Lassar, A. B. Wnt signals from the neural tube block ectopic cardiogenesis. Genes Dev. 15, 255-260 (2001).

25. Mukhopadhyay, M. et al. Dickkopf1 is required for embryonic head induction and limb morphogenesis in the mouse. Dev. Cell 1, 423-434 (2001).

26. Kobayashi, K. et al. Secreted Frizzled-related protein 2 is a procollagen $\mathrm{C}$ proteinase enhancer with a role in fibrosis associated with myocardial infarction. Nature Cell Biol. 11, 46-55 (2009).

27. He, W. et al. Exogenously administered secreted frizzled related protein 2 (Sfrp2) reduces fibrosis and improves cardiac function in a rat model of myocardial infarction. Proc. Natl Acad. Sci. USA 107, 21110-21115 (2010).

28. Kawano, Y. \& Kypta, R. Secreted antagonists of the Wnt signalling pathway. J. Cell Sci. 116, 2627-2634 (2003).

29. Bergmann, M. W. WNT signaling in adult cardiac hypertrophy and remodeling: lessons learned from cardiac development. Circ. Res. 107, 1198-1208 (2010).

30. Liang, X. et al. HCN4 dynamically marks the first heart field and conduction system precursors. Circ. Res. 113, 399-407 (2013).

31. Soriano, P. Generalized lacZ expression with the ROSA26 Cre reporter strain. Nature Genet. 21, 70-71 (1999).

32. Laugwitz, K. L., Moretti, A., Caron, L., Nakano, A. \& Chien, K. R. Islet1 cardiovascular progenitors: a single source for heart lineages? Development 135, 193-205 (2008)

33. Moretti, A. et al. Multipotent embryonic Isl1 ${ }^{+}$progenitor cells lead to cardiac, smooth muscle, and endothelial cell diversification. Cell 127, 1151-1165 (2006).
34. Spater, D. et al. A HCN4 + cardiomyogenic progenitor derived from the first heart field and human pluripotent stem cells. Nature Cell Biol. 15, 1098-1106 (2013).

35. Bruneau, B. G. et al. A murine model of Holt-Oram syndrome defines roles of the T-box transcription factor Tbx 5 in cardiogenesis and disease. Cell 106, 709-721 (2001).

36. Devine, W. P., Wythe, J. D., George, M., Koshiba-Takeuchi, K. \& Bruneau, B. G. Early patterning and specification of cardiac progenitors in gastrulating mesoderm. eLife 3, e03848 (2014).

37. Kokkinopoulos, I. et al. Single-cell expression profiling reveals a dynamic state of cardiac precursor cells in the early mouse embryo. PLoS ONE 10, e0140831 (2015).

38. Yamagishi, H. et al. Tbxl is regulated by tissue-specific forkhead proteins through a common Sonic hedgehog-responsive enhancer. Genes Dev. 17, 269-281 (2003).

39. Huynh, T., Chen, L., Terrell, P. \& Baldini, A. A fate map of Tbx1 expressing cells reveals heterogeneity in the second cardiac field. Genesis 45, 470-475 (2007).

40. Satoh, W., Matsuyama, M., Takemura, H., Aizawa, S. \& Shimono, A. Sfrp1, Sfrp2, and Sfrp5 regulate the Wnt/beta-catenin and the planar cell polarity pathways during early trunk formation in mouse. Genesis 46, 92-103 (2008).

41. Stevens, S. M. \& Pu, W. T. HCN4 charges up the first heart field. Circ. Res. 113, 350-351 (2013)

42. Mikawa, T. \& Gourdie, R. G. Pericardial mesoderm generates a population of coronary smooth muscle cells migrating into the heart along with ingrowth of the epicardial organ. Dev. Biol. 174, 221-232 (1996).

43. Pennisi, D. J., Rentschler, S., Gourdie, R. G., Fishman, G. I. \& Mikawa, T. Induction and patterning of the cardiac conduction system. Int. J. Dev. Biol. 46, 765-775 (2002).

44. Asai, R. et al. Endothelin receptor type A expression defines a distinct cardiac subdomain within the heart field and is later implicated in chamber myocardium formation. Development 137, 3823-3833 (2010).

45. Kapoor, N., Liang, W., Marban, E. \& Cho, H. C. Direct conversion of quiescent cardiomyocytes to pacemaker cells by expression of Tbx18. Nature Biotechnol. 31, 54-62 (2013)

46. Greulich, F. et al. Misexpression of Tbx18 in cardiac chambers of fetal mice interferes with chamber-specific developmental programs but does not induce a pacemaker-like gene signature. J. Mol. Cell. Cardiol. 97, 140-149 (2016).

47. Lescroart, F. et al. Clonal analysis reveals common lineage relationships between head muscles and second heart field derivatives in the mouse embryo. Development 137, 3269-3279 (2010).

48. Zaffran, S., Kelly, R. G., Meilhac, S. M., Buckingham, M. E. \& Brown, N. A. Right ventricular myocardium derives from the anterior heart field. Circ. Res. 95, 261-268 (2004)

49. Kelly, R. G. Building the right ventricle. Circ. Res. 100, 943-945 (2007).

50. Jensen, B., Moorman, A. F. \& Wang, T. Structure and function of the hearts of lizards and snakes. Biol. Rev. Camb. Philos. Soc. 89, 302-336 (2014).

51. Klaus, A., Saga, Y., Taketo, M. M., Tzahor, E. \& Birchmeier, W. Distinct roles of Wnt/beta-catenin and Bmp signaling during early cardiogenesis. Proc. Natl Acad. Sci. USA 104, 18531-18536 (2007).

52. Norden, J., Greulich, F., Rudat, C., Taketo, M. M. \& Kispert, A. Wnt/betacatenin signaling maintains the mesenchymal precursor pool for murine sinus horn formation. Circ. Res. 109, e42-e50 (2011).

53. Kawamoto, S. et al. A novel reporter mouse strain that expresses enhanced green fluorescent protein upon Cre-mediated recombination. FEBS Lett. 470, 263-268 (2000).

\section{Acknowledgements}

We thank Mr Hidemichi Miyahara and Mr Makoto Kiso for their technical assistance, Dr Rieko Asai, Dr Hiroki Kurihara and Dr Hiroyuki Yamagishi for sharing probes for in situ hybridization, Dr Kenta Yashiro for helpful discussions and suggestions and all members of Saga's and Yoshizumi's laboratories. We also thank the Natural Science Centre for Basic Research and Development (N-BARD) at Hiroshima University for allowing the use of their facility. This study was supported in part by a Grant-in-Aid for Scientific Research from the Japanese Ministry of Education, Culture, Sports, Science, and Technology (22590183 and 16K08443). This work was also supported by NIG-JOINT (2012-B, 2013-B and 2016-A1)

\section{Author contributions}

M.F. and A.S. performed the experiments. R.K., M.N., U.K., S.M.E., M.Y., A.S., Y.S. and H.K supplied the experimental apparatus and animals. All authors contributed to the analyses of the experimental data. M.Y, A.S., Y.S. and H.K. designed the research. M.F., A.S., Y.S and H.K. wrote the manuscript. 


\section{Additional information}

Supplementary Information accompanies this paper at http://www.nature.com/ naturecommunications

Competing financial interests: The authors declare no competing financial interests.

Reprints and permission information is available online at http://npg.nature.com/ reprintsandpermissions/

How to cite this article: Fujii, M. et al. Sfrp5 identifies murine cardiac progenitors for all myocardial structures except for the right ventricle. Nat. Commun. 8, 14664 doi: 10.1038/ncomms14664 (2017).
Publisher's note: Springer Nature remains neutral with regard to jurisdictional claims in published maps and institutional affiliations.

(c) (i) This work is licensed under a Creative Commons Attribution 4.0 International License. The images or other third party material in this article are included in the article's Creative Commons license, unless indicated otherwise in the credit line; if the material is not included under the Creative Commons license, users will need to obtain permission from the license holder to reproduce the material. To view a copy of this license, visit http://creativecommons.org/licenses/by/4.0/

(C) The Author(s) 2017 